\title{
Sensitivity Analysis of Multidisciplinary Rotorcraft Simulations
}

\author{
Li Wang * Boris Diskin $^{\dagger}$ \\ National Institute of Aerospace, Hampton, VA, USA \\ Robert T. Biedron ${ }^{\ddagger}$ Eric J. Nielsen $§$ \\ NASA Langley Research Center, Hampton, VA, USA \\ Olivier A. Bauchau II \\ University of Maryland, MD, USA
}

\begin{abstract}
A multidisciplinary sensitivity analysis of rotorcraft simulations involving tightly coupled high-fidelity computational fluid dynamics and comprehensive analysis solvers is presented and evaluated. An unstructured sensitivity-enabled Navier-Stokes solver, FUN3D, and a nonlinear flexible multibody dynamics solver, DYMORE, are coupled to predict the aerodynamic loads and structural responses of helicopter rotor blades. A discretely-consistent adjoint-based sensitivity analysis available in FUN3D provides sensitivities arising from unsteady turbulent flows and unstructured dynamic overset meshes, while a complex-variable approach is used to compute DYMORE structural sensitivities with respect to aerodynamic loads. The multidisciplinary sensitivity analysis is conducted through integrating the sensitivity components from each discipline of the coupled system. Numerical results verify accuracy of the FUN3D/DYMORE system by conducting simulations for a benchmark rotorcraft test model and comparing solutions with established analyses and experimental data. Complex-variable implementation of sensitivity analysis of DYMORE and the coupled FUN3D/DYMORE system is verified by comparing with real-valued analysis and sensitivities. Correctness of adjoint formulations for FUN3D/DYMORE interfaces is verified by comparing adjoint-based and complex-variable sensitivities. Finally, sensitivities of the lift and drag functions obtained by complex-variable FUN3D/DYMORE simulations are compared with sensitivities computed by the multidisciplinary sensitivity analysis, which couples adjoint-based flow and grid sensitivities of FUN3D and FUN3D/DYMORE interfaces with complex-variable sensitivities of DYMORE structural responses.
\end{abstract}

\section{Introduction}

High-fidelity analysis methods for rotorcraft aeromechanics have become increasingly important to capture complex physics involving transonic flows, vortical wakes, reversed flows, blade vortex interactions (BVI), and large amplitudes of elastic flapping, lagging and torsion motions. A rotorcraft analysis often requires many disciplines such as aerodynamics, aeroacoustics, structural dynamics and deformations, flight mechanics, and others to account for complex interactions of unsteady fluids with highly flexible rotor blades. Multidisciplinary rotorcraft comprehensive analysis (CA) tools ${ }^{1-4}$ are often used to simulate rotorcraft aeromechanics. These tools are fast and comprehensive, but rely on low-fidelity aerodynamic models, such as lifting line and vortex wake models. The state of the art in highfidelity rotorcraft analysis is represented by simulations that couple a CA code with a physics-based, first-principle computational fluid dynamics (CFD) code. ${ }^{5-7}$

Gradient-based optimization of rotorcraft simulations has been a focus of intensive studies in the past 30 years. ${ }^{8-10}$ Most of the studies involving CA tools have been conducted using finite-difference approximations for sensitivities. Finite-difference approaches are effective for computing sensitivity of many objective functions with respect to a few design parameters. The number of simulations required for such computations is proportional to the number of design parameters and may be numbered in hundreds for design and optimization of complex rotorcraft configurations. Finitedifference optimization approach may be acceptable for optimization of non-expensive CA models, but it is not feasible

\footnotetext{
*Senior Research Engineer, AIAA Member, li.wang@ nianet.org

${ }^{\dagger}$ Research Fellow, AIAA Associate Fellow, bdiskin@nianet.org

₹esearch Scientist, robert.t.biedron@nasa.gov

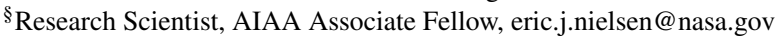

IProfessor, AIAA Senior Member, obauchau@umd.edu
} 
for high-fidelity rotorcraft analysis, in which a single large-scale simulation involving a coupled CFD/CA system may require hundreds of thousands of CPU hours of high-performance computing. On the other hand, adjoint formulations developed in some state of the art CFD solvers enable simultaneous computations of sensitivity of an objective function to many design parameters. ${ }^{11-14}$ The computational cost of an adjoint-based analysis approximately equals to the cost of a single simulation and does not increase with the number of design parameters. This property makes adjoint methods especially suitable for shape optimization where a single or a few objective functions are used, but the number of design parameters is large. Discretely consistent adjoint methods offer an additional advantage that the computed numerical sensitivities can be rigorously verified through comparisons with the exact sensitivities computed by a complex-variable approach. ${ }^{15}$ In contrast with the finite-difference methods employing real-valued perturbations, the complex-variable approach does not suffer from subtractive error cancellation and is capable of producing the true sensitivity of the discrete solution with respect to a chosen design parameter. Recently, adjoint-based sensitivity analyses of model rotorcraft simulations ${ }^{11,16,17}$ have been demonstrated to guide design and optimization.

In the context of multidisciplinary simulations, the sensitivity analysis and the subsequent gradient-based optimization procedures must include sensitivities across all disciplines, thereby leading to a multidisciplinary sensitivity analysis. While discretely-consistent adjoint-based multidisciplinary sensitivity analysis is the ultimate goal, the implementation of a discretely-consistent formulation in a production-level code is a complex task that may require years of development effort. Moreover, development of a multidisciplinary adjoint formulation requires detailed expertise and unlimited access to the source codes in each discipline involved. Modern multidisciplinary applications often couple large legacy codes developed over many years by different groups and organizations. The codes often exchange data through specialized interfaces without exposing the internal treatment of the discipline solutions. In many cases, the source codes and/or expertise related to a particular discipline are simply not available. Even if the required expertise and source codes are available, the computational time may not be evenly distributed between disciplines. A useful multidisciplinary sensitivity analysis tool can couple efficient adjoint-based sensitivities computed for "heavier" disciplines such as CFD with sensitivities computed by "black-box" or "grey-box" methods for "lighter" disciplines such as CA. The "black-box" methods may involve real or complex-valued finite-difference approaches; the "greybox" methods may involve automatic differentiation methods. ${ }^{18,19}$ The sensitivity analysis for each discipline can then be integrated within a multidisciplinary sensitivity analysis framework. A theoretical foundation for such integrations has been presented in the literature. ${ }^{20}$

The goal of this paper is to present and evaluate a multidisciplinary sensitivity analysis approach for high-fidelity CFD/CA coupled simulations based on a tight coupling methodology. An unstructured, highly-scalable CFD solver, FUN3D, ${ }^{6,21,22}$ and a nonlinear flexible multibody dynamics CA code, DYMORE, ${ }^{1}$ are coupled to predict the rotor airloads and structural responses of helicopter rotor blades. For overset meshes, the domain-connectivity information is established via the software libraries described in Ref. 23. A discretely-consistent adjoint-based sensitivity analysis available in FUN3D provides sensitivities arising from unsteady turbulent flows and unstructured dynamic overset meshes, while a complex-variable approach is used to compute DYMORE structural sensitivities with respect to aerodynamic loads. In contrast to the analysis procedure, in which DYMORE operates in the serial execution mode, the sensitivity analysis of DYMORE is distributed to all the processors used in the CFD analysis. The computations of sensitivity of structural responses with respect to different airload components are embarrassingly parallel and can be simultaneously conducted by different processors. The multidisciplinary sensitivity analysis integrates all sensitivity components from the coupled systems.

The material of the paper is presented as follows. Section II highlights the technical approach used in this paper. A mathematical formulation for adjoint-based sensitivities of FUN3D coupled with complex-variable sensitivities for DYMORE is presented. Section III provides verification studies for the current implementation of the FUN3D/DYMORE (F/D) coupled analysis. The complex-variable analyses for the DYMORE model and the multidisciplinary F/D model are verified. The adjoint formulations for F/D interfaces and multidisciplinary sensitivity analysis are examined and verified by comparing with real-valued finite-difference and complex-variable sensitivities. Finally, Section IV summarizes the work and discusses future plans.

\section{Technical Approach}

In this section, the CFD and CA solvers used in this study are first outlined, followed by a brief description of the CFD/CA coupling interfaces and the loose and tight coupling procedures. A complex-variable approach for computing sensitivities is discussed, and a mathematical formulation of the multidisciplinary CFD/CA sensitivity 
analysis is given.

\section{A. Flow and Comprehensive Analysis Solvers}

Solutions of the Reynolds-averaged Navier-Stokes (RANS) equations are computed with the FUN3D flow solver, $6,21,22$ developed and supported by NASA Langley Research Center. A standard one-equation Spalart-Allmaras turbulence model $^{24}$ is used for the current simulations. FUN3D is a finite-volume, node-centered, unstructured-grid RANS solver, which is widely used for high-fidelity analysis and adjoint-based design of complex turbulent flows. ${ }^{25,26}$ FUN3D solves the governing flow equations on mixed-element grids; the elements can be tetrahedra, pyramids, prisms, and hexahedra. At median-dual control-volume faces, the inviscid fluxes are computed using an approximate Riemann solver. In the current study, Roe's flux difference splitting ${ }^{27}$ is used. For second-order accuracy, solutions at dual faces are obtained by a UMUSCL (Unstructured Monotonic Upstream-centered Scheme for Conservation Laws) scheme ${ }^{28,29}$ with the coefficient set to be $\kappa=0.5$ for the meanflow equations and $\kappa=0$ for the turbulence model. The viscous fluxes are discretized with a finite-volume formulation, in which the Green-Gauss theorem is used to compute gradients on the dual faces for tetrahedral meshes; this is equivalent to a Galerkin type approximation. For nontetrahedral meshes, the Green-Gauss (cell-based) gradients are combined with edge-based gradients to improve the $h$-ellipticity of viscous operators. The diffusion term in the turbulence model is handled in the same fashion as the meanflow viscous terms. The vorticity-based source term for the turbulence model is computed using velocity gradients evaluated by the Green-Gauss method on dual volumes. To advance the equations in time, a blended second- and third-order backward-difference scheme, referred to as $\mathrm{BDF} 2{ }_{\text {opt }},{ }^{30}$ is employed; the scheme is second-order accurate in time, but has a smaller leading error term than the standard second-order backward difference scheme (BDF2). For overset meshes, the DiRTlib ${ }^{31}$ and SUGGAR $++^{23}$ codes are used to facilitate communications between components of the mesh.

The CA code used in this study is DYMORE, ${ }^{1}$ a nonlinear flexible multibody dynamics analysis code that provides static, dynamic, stability, and trim analyses of rotorcraft configurations. DYMORE contains libraries of primitive elements such as rigid bodies, mechanical joints, elastic springs, dampers, beams, shells and plates. For computational structural dynamics analysis, DYMORE uses a high-fidelity finite-element method in the time domain without relying on a low-fidelity model reduction method. Although DYMORE has not been developed specifically for rotorcraft applications, it has been widely used in this area due to its capabilities of handling nonlinear flexible systems with arbitrary topologies. The internal aerodynamic model in DYMORE is a low fidelity approximation based on lifting line theory and vortex wake models. To enable sensitivity computations conducted via a complex-variable approach, a complex-variable version of DYMORE has been developed and verified by comparing with real-valued analyses.

\section{B. CFD/CA Coupling Approaches}

An F/D interface has been previously developed to enable the exchange of aerodynamic loads and structural deflections between high-fidelity aero and structural dynamics models. ${ }^{32}$ In the current work, the interface has been extended to handle mixed-mode communications between real-mode FUN3D and complex-mode DYMORE as well as to enable multidisciplinary sensitivity analysis. The new implementation of the F/D system can conduct loose or tight coupling simulations.

In a loose coupling approach, ${ }^{33}$ an initial solution from DYMORE, using its internal linear aerodynamics model, provides an initial estimate of the elastic blade deformations for FUN3D. CFD computations start from a freestream flow; delta airloads between the CFD solution and lifting-line computation in DYMORE are collected along with the time advancement process for one revolution in the first coupling iteration and $2 / N_{b}$ revolutions in the subsequent coupling iterations. Here, $N_{b}$ denotes the number of rotor blades. The use of one full revolution in the first coupling iteration aims to reduce the transient effects caused by freestream initial conditions. Once the computation of the flow field is complete, the delta airloads are passed to DYMORE to compute trimmed elastic motions, which are transferred back to FUN3D to generate new blade surface geometries and motions. This process is repeated until a converged periodic solution is achieved.

In a tight coupling approach, the CFD and CA models are still executed separately, but the data exchange occurs once per time step. Fig. 1 shows a diagram illustrating the F/D tight coupling procedures, in which FUN3D functions that have DYMORE structural solutions as inputs are highlighted in red. At each coupling iteration, DYMORE performs one time-step advancement with a set of sectional airloads as inputs. The structural solutions describing linear and angular displacements at the quarter-chord line of rotor blades are sent to FUN3D. High-order surface spline functions are created, followed by an extraction of average rigid motions from the CA displacements and a reposition of the CFD surface grid. The CFD volume grid is rigidly moved and elastically deformed to accommodate 


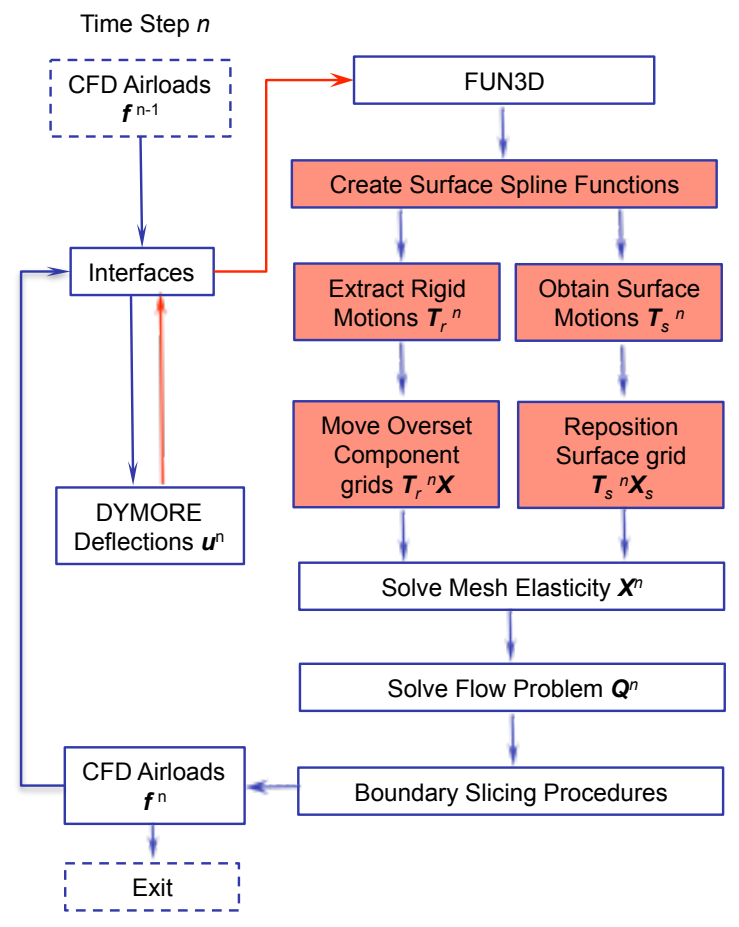

Figure 1. Diagram illustrating the tight coupling procedure of F/D models.

the new surface mesh. The CFD flow problem is solved on the updated mesh. When a desired convergence level is reached, a boundary slicing procedure is performed to obtain updated sectional airloads (forces and moments) at specified airstation locations. The new CFD airloads are then transferred to DYMORE through the interface to start the next coupling iteration. In the present implementation of the tight coupling procedures, the data exchange between the CFD and CA models is performed in memory by linking FUN3D with a prebuilt DYMORE library, which results in direct, fast data access. For verification purposes, the tight coupling computation is conducted with trim control angles that have been obtained from a loose coupling procedure. The trim control parameters are not updated during the tight coupling analysis procedure, and therefore trim computations are avoided. This simplification significantly accelerates the iterative convergence of DYMORE solutions. In future design and optimization applications, the trim control parameters will be updated through constraint optimization procedures. ${ }^{16,25}$ In Section III.A, a verification study is conducted to demonstrate the accuracy of the current F/D loose and tight coupling procedures for the HARTII baseline rotorcraft configuration. ${ }^{7}$

\section{Complex-Variable Approach}

In this work, the complex-variable method ${ }^{15}$ is employed by the multidisciplinary CFD/CA sensitivity analysis to compute sensitivities of structural responses with respect to aerodynamic loads and to verify multidisciplinary sensitivities. A brief description of the method is given as follows.

For a real-valued function, $f(x)$, of a real variable $x$, the complex-variable method allows simultaneous evaluation of the function and its derivative, $d f / d x$. For an input, $x+i h$, with an imaginary perturbation, $i h$, the complex-valued function can be expanded in a Taylor series as:

$$
f(x+i h)=f(x)+i h \frac{d f}{d x}-\frac{h^{2}}{2} \frac{d^{2} f}{d x^{2}}-i \frac{h^{3}}{6} \frac{d^{3} f}{d x^{3}}+\cdots .
$$

From this equation, the real part of the complex-valued expansion gives the function itself,

$$
f(x) \approx \operatorname{Re}[f(x+i h)],
$$

and the imaginary part yields the derivative

$$
\frac{d f}{d x} \approx \frac{\operatorname{Im}[f(x+i h)]}{h},
$$


with a truncation error $O\left(h^{2}\right)$.

Unlike a finite-difference method with a real-valued perturbation, the complex-variable approach does not suffer from subtractive cancellation errors, thereby providing increased accuracy and robustness. Within machine accuracy, derivatives computed by the complex-variable method with perturbation $h=10^{-50}$ can be considered as the true derivatives with zero truncation error.

The standard FUN3D software suite allows for complex-mode operation to verify hand-differentiated sensitivities, but a similar capability was not available in DYMORE. For the present study, a complex mode has been developed in DYMORE by replacing all double and float variables declared in the original (i.e., real-valued) DYMORE code with complex variables of double precision and redefining operators needed for complex-valued operations. The complex mode of DYMORE enables the evaluation of comprehensive analysis sensitivities via the complex-variable approach. The F/D interface has been extended to operate in the complex mode, supporting the exchange of complex-valued aerodynamic forces, moments and surface displacements.

\section{Sensitivity Analysis}

A mathematical formulation for the sensitivities of a tight coupling multidisciplinary CFD/CA analysis is given in this section. The following notations are used: $\mathbf{u}^{n}$ is a CA solution vector at a given time level $n$. The vector is used to update the surface position and the average rigid motion of component CFD grids at time level $n$. Initial CA solution, $\mathbf{u}^{0}$, is given as a solution of a loose coupling formulation and is independent of design parameters. A vector of airloads, $\mathbf{f}^{n}$, is computed by the CFD code at time level $n$ at specified airstations. The fixed baseline volume and surface grids, $\overline{\mathbf{X}}$ and $\overline{\mathbf{X}}_{s}$, are generated based on the baseline blade configuration, independent of design parameters, and used to specify the baseline elasticity matrix, $\overline{\mathbf{K}}$,

$$
\overline{\mathbf{K}} \overline{\mathbf{X}}=\overline{\mathbf{X}}_{s} .
$$

The reference component volume and surface grids, $\hat{\mathbf{X}}$ and $\hat{\mathbf{X}}_{s}$, account for variations of shape design parameters (used to perturb the surface geometry), $\mathbf{D}$, and define the reference frame. The reference blade grid $\hat{\mathbf{X}}$ is computed by solving a mesh elasticity problem

$$
\overline{\mathbf{K}} \hat{\mathbf{X}}=\hat{\mathbf{X}}_{s}(\mathbf{D}) .
$$

The overset volume and surface grids, $\mathbf{X}^{n}$ and $\mathbf{X}_{s}^{n}$, reflect the actual positions of all blades at time level $n$. The initial grid, $\mathbf{X}^{0}$, corresponding to time level $n=0$ is computed by rigidly moving the reference blade grid to the corresponding initial position for each blade and combining these component grids with a stationary background grid.

$$
\begin{gathered}
\mathbf{X}^{0}=\overline{\mathbf{T}}_{r}^{0} \hat{\mathbf{X}}, \\
\overline{\mathbf{T}}_{r}^{0}=\overline{\mathbf{T}}^{0}\left(\overline{\boldsymbol{\delta}}\left(\mathbf{u}^{0}\right), \bar{\beta}\left(\mathbf{u}^{0}\right), \bar{\theta}\left(\mathbf{u}^{0}\right)\right) \mathbf{T}_{\phi_{0}} .
\end{gathered}
$$

Here, $\delta, \beta$, and $\theta$ are lead-lag, flap, and pitch control angles, respectively; $\bar{\delta}, \bar{\beta}$, and $\bar{\theta}$ denote quantities averaged over the entire span of a rotor blade; $\overline{\mathbf{T}}^{0}\left(\bar{\delta}\left(\mathbf{u}^{0}\right), \bar{\beta}\left(\mathbf{u}^{0}\right), \bar{\theta}\left(\mathbf{u}^{0}\right)\right)$ is an extracted (composite) rigid motion corresponding to averaged CA displacements. As described in Ref. 32, this averaged rigid motion helps keep the elastically-deflected blade centered within the blade volume mesh, and helps prevent negative volumes during the mesh deformation process, when large flapping motions are present. The transform matrix for the composite rigid motion is obtained by successive multiplications of the transform matrices corresponding to each rigid motion,

$$
\overline{\mathbf{T}}(\bar{\delta}, \bar{\beta}, \bar{\theta})=\mathbf{T}(\bar{\delta}) \cdot \mathbf{T}(\bar{\beta}) \cdot \mathbf{T}(\bar{\theta}) .
$$

Each rigid motion is represented as a $4 \times 4$ matrix

$$
\mathbf{T}=\left[\begin{array}{cccc}
R_{11} & R_{12} & R_{13} & t_{x} \\
R_{21} & R_{22} & R_{23} & t_{y} \\
R_{31} & R_{32} & R_{33} & t_{z} \\
0 & 0 & 0 & 1
\end{array}\right] .
$$

Here, the $3 \times 3$ block matrix $\mathbf{R}$ defines a general rotation and the vector $\mathbf{t}$ appearing in the fourth column of the transform matrix defines a translation. The motion $\mathbf{T}_{\phi_{0}}$ in Eq. (7) is a rigid motion transforming a blade from its initial 
position to the reference frame, in which the CA displacements are defined. The grid equations at times $n \geq 1$ include elastic grid deformations satisfying

$$
\begin{gathered}
\mathbf{K}^{n} \mathbf{X}^{n}=\mathbf{T}_{s}^{n} \hat{\mathbf{X}}_{s}(\mathbf{D}), \\
\mathbf{T}_{s}^{n}=\mathbf{T}_{\psi}^{n} \mathbf{T}^{n}\left(\boldsymbol{\delta}\left(\mathbf{u}^{n}\right), \beta\left(\mathbf{u}^{n}\right), \theta\left(\mathbf{u}^{n}\right)\right) \mathbf{T}_{\phi_{0}}
\end{gathered}
$$

Here, $\mathbf{T}_{s}$ denotes the elastic motions applied to individual surface nodes, and $\mathbf{T}_{\psi}$ is a rigid motion corresponding to the azimuthal rotation of the blades. The elasticity matrix, $\mathbf{K}^{n}$, is derived from a rigidly moved reference grid, satisfying:

$$
\begin{gathered}
\mathbf{K}^{n} \overline{\mathbf{T}}_{r}^{n} \hat{\mathbf{X}}=\overline{\mathbf{T}}_{r}^{n} \hat{\mathbf{X}}_{s}, \\
\overline{\mathbf{T}}_{r}^{n}=\mathbf{T}_{\psi}^{n} \overline{\mathbf{T}}^{n}\left(\overline{\boldsymbol{\delta}}\left(\mathbf{u}^{n}\right), \bar{\beta}\left(\mathbf{u}^{n}\right), \bar{\theta}\left(\mathbf{u}^{n}\right)\right) \mathbf{T}_{\phi_{0}} .
\end{gathered}
$$

Note that the elasticity matrix at time level $n$ relates to the baseline elasticity matrix as

$$
\mathbf{K}^{n} \overline{\mathbf{T}}_{r}^{n}=\overline{\mathbf{T}}_{r}^{n} \overline{\mathbf{K}}
$$

The vector $\mathbf{Q}^{n}$ is a vector of CFD solutions at time level $n$. The solution at time $n=0$ is specified from a precomputed loose coupling solution, $\overline{\mathbf{Q}}$, that may depend on CFD design parameters such as angle of attack, Mach number, etc. Given variables that are independent of design parameters, $\overline{\mathbf{K}}, \mathbf{u}^{0}$, and $\mathbf{T}_{\phi_{0}}$, and variables that may depend on design parameters, $\overline{\mathbf{Q}}(\mathbf{D}), \hat{\mathbf{X}}_{s}(\mathbf{D})$, and $\mathbf{T}_{\psi}(\mathbf{D})$, the following equations for a tight coupling formulation are written in the order in which the equations are solved:

$$
\begin{array}{rlll}
\text { Reference Grid : } & \hat{\mathbf{G}}\left(\hat{\mathbf{X}}, \hat{\mathbf{X}}_{s}, \mathbf{D}\right) & \equiv \overline{\mathbf{K}} \hat{\mathbf{X}}-\hat{\mathbf{X}}_{s}(\mathbf{D})=\mathbf{0}, & \\
\text { Initial Grid : } & \mathbf{G}^{0}\left(\mathbf{X}^{0}, \hat{\mathbf{X}}, \overline{\mathbf{T}}_{r}^{0}\left(\mathbf{u}^{0}\right), \mathbf{D}\right) & \equiv \mathbf{X}^{0}-\overline{\mathbf{T}}_{r}^{0} \hat{\mathbf{X}}=\mathbf{0}, & \\
\text { Initial Flow : } & \mathbf{R}^{0}\left(\mathbf{Q}^{0}, \overline{\mathbf{Q}}(\mathbf{D})\right) & \equiv \mathbf{Q}^{0}-\overline{\mathbf{Q}}(\mathbf{D})=\mathbf{0}, & \\
\text { Initial Load : } & \mathbf{F}^{0}\left(\mathbf{f}^{0}, \mathbf{Q}^{0}, \mathbf{X}^{0}\right) & =\mathbf{0}, & (1 \leq n \leq N) \\
\text { CA : } & \mathbf{C}^{n}\left(\mathbf{u}^{n}, \mathbf{u}^{n-1}, \mathbf{f}^{n-1}, \mathbf{D}\right) & =\mathbf{0}, & (1 \leq n \leq N) \\
\text { CFD Grid : } & \mathbf{G}^{n}\left(\mathbf{X}^{n}, \hat{\mathbf{X}}_{s}, \overline{\mathbf{T}}_{r}^{n}\left(\mathbf{u}^{n}\right), \mathbf{T}_{s}^{n}\left(\mathbf{u}^{n}\right), \mathbf{D}\right) & \equiv \overline{\mathbf{T}}_{r}^{n} \overline{\mathbf{K}}\left(\overline{\mathbf{T}}_{r}^{n}\right)^{-1} \mathbf{X}^{n}-\mathbf{T}_{s}^{n} \hat{\mathbf{X}}_{s}(\mathbf{D})=\mathbf{0}, & \\
\text { CFD Flow : } & \mathbf{R}^{n}\left(\mathbf{Q}^{n}, \mathbf{Q}^{n-1}, \mathbf{X}^{n}, \mathbf{X}^{n-1}, \mathbf{D}\right) & =\mathbf{0}, & (1 \leq n \leq N) \\
\text { Loads : } & \mathbf{F}^{n}\left(\mathbf{f}^{n}, \mathbf{Q}^{n}, \mathbf{X}^{n}\right) & =\mathbf{f}^{n}-\mathbf{S}^{n}\left(\mathbf{Q}^{n}, \mathbf{X}^{n}\right)=\mathbf{0} . &
\end{array}
$$

Here, $\mathbf{S}$ denotes a boundary slicing function performed in FUN3D for obtaining sectional airloads. For simplicity of presentation, the time-dependent equations are assumed to depend on solutions at the current and one preceding time level only. In the actual solver, the time derivatives are discretized through second-order backward difference schemes that use solutions at the current and up to three preceding time levels. The residuals of the CFD equations are in the form of an Arbitrary Eulerian-Lagrangian formulation that is suitable for dynamic deformable grids and accounts for grid speeds and geometric conservation law. The details of CFD equations on overset grids are provided in Ref. 25.

In the sensitivity analysis framework presented here, the CFD flow and grid equations and the loads equations use adjoint formulations, whereas sensitivities of the surface-grid elastic motion $\mathbf{T}_{s}$ and the extracted rigid motion $\overline{\mathbf{T}}_{r}$ to the aerodynamic loads $\mathbf{f}^{n}$ are computed by the complex-variable method. For a general objective function $g(\mathbf{Q}, \mathbf{u}, \mathbf{X}, \mathbf{f}, \mathbf{D})$, the adjoint formulation is derived via the Lagrangian functional formed as

$$
L=g(\mathbf{Q}, \mathbf{u}, \mathbf{X}, \mathbf{f}, \mathbf{D})+\sum_{n=0}^{N}\left[\boldsymbol{\Lambda}_{R}^{n}\right]^{T} \mathbf{R}^{n}+\sum_{n=0}^{N}\left[\boldsymbol{\Lambda}_{G}^{n}\right]^{T} \mathbf{G}^{n}+\sum_{n=0}^{N}\left[\boldsymbol{\Lambda}_{F}^{n}\right]^{T} \mathbf{F}^{n}+\left[\boldsymbol{\Lambda}_{\hat{G}}\right]^{T} \hat{\mathbf{G}}
$$

where $\mathbf{Q}, \mathbf{u}, \mathbf{X}$, and $\mathbf{f}$ represent CFD solutions, CA solutions, grid, and load solutions at all time steps, respectively; $\boldsymbol{\Lambda}_{R}$, $\boldsymbol{\Lambda}_{G}$, and $\boldsymbol{\Lambda}_{F}$ are the vectors of the time-dependent adjoint solutions for the flow, grid and loads equations, respectively; $\boldsymbol{\Lambda}_{\hat{G}}$ denotes the adjoint solution for the reference grid; and the superscript $T$ represents the transposition operator. Differentiating the Lagrangian with respect to design variables $\mathbf{D}$ and equating the coefficients of state variable sensitivities to zero ${ }^{34}$ yields the following adjoint equations: 
for $n=N$,

$$
\begin{array}{lll}
\text { Load : } & {\left[\frac{\partial g}{\partial \mathbf{f}^{N}}\right]+\left[\boldsymbol{\Lambda}_{F}^{N}\right]^{T}\left[\frac{\partial \mathbf{F}^{N}}{\partial \mathbf{f}^{N}}\right]} & =0 \\
\text { Flow : } & {\left[\frac{\partial g}{\partial \mathbf{Q}^{N}}\right]+\left[\boldsymbol{\Lambda}_{R}^{N}\right]^{T}\left[\frac{\partial \mathbf{R}^{N}}{\partial \mathbf{Q}^{N}}\right]+\left[\boldsymbol{\Lambda}_{F}^{N}\right]^{T}\left[\frac{\partial \mathbf{F}^{N}}{\partial \mathbf{Q}^{N}}\right]} & =0 \\
\text { Grid : } & {\left[\frac{\partial g}{\partial \mathbf{X}^{N}}\right]+\left[\boldsymbol{\Lambda}_{G}^{N}\right]^{T}\left[\frac{\partial \mathbf{G}^{N}}{\partial \mathbf{X}^{N}}\right]+\left[\boldsymbol{\Lambda}_{R}^{N}\right]^{T}\left[\frac{\partial \mathbf{R}^{N}}{\partial \mathbf{X}^{N}}\right]+\left[\boldsymbol{\Lambda}_{F}^{N}\right]^{T}\left[\frac{\partial \mathbf{F}^{N}}{\partial \mathbf{X}^{N}}\right]} & =0
\end{array}
$$

for $1 \leq n<N$,

$$
\begin{array}{lll}
\text { Load : } & {\left[\frac{\partial g}{\partial \mathbf{f}^{n}}\right]+\left[\boldsymbol{\Lambda}_{F}^{n}\right]^{T}\left[\frac{\partial \mathbf{F}^{n}}{\partial \mathbf{f}^{n}}\right]+\sum_{k=n+1}^{N}\left[\boldsymbol{\Lambda}_{G}^{k}\right]^{T}\left(\left[\frac{\partial \mathbf{G}^{k}}{\partial \overline{\mathbf{T}}_{r}^{k}}\right]\left[\frac{\partial \overline{\mathbf{T}}_{r}^{k}}{\partial \mathbf{f}^{n}}\right]+\left[\frac{\partial \mathbf{G}^{k}}{\partial \mathbf{T}_{S}^{k}}\right]\left[\frac{\partial \mathbf{T}_{s}^{k}}{\partial \mathbf{f}^{n}}\right]\right)} & =0, \\
\text { Flow : } & {\left[\frac{\partial g}{\partial \mathbf{Q}^{n}}\right]+\left[\boldsymbol{\Lambda}_{R}^{n}\right]^{T}\left[\frac{\partial \mathbf{R}^{n}}{\partial \mathbf{Q}^{n}}\right]+\left[\boldsymbol{\Lambda}_{R}^{n+1}\right]^{T}\left[\frac{\partial \mathbf{R}^{n+1}}{\partial \mathbf{Q}^{n}}\right]+\left[\boldsymbol{\Lambda}_{F}^{n}\right]^{T}\left[\frac{\partial \mathbf{F}^{n}}{\partial \mathbf{Q}^{n}}\right]} & =0, \\
\text { Grid : } & {\left[\frac{\partial g}{\partial \mathbf{X}^{n}}\right]+\left[\boldsymbol{\Lambda}_{G}^{n}\right]^{T}\left[\frac{\partial \mathbf{G}^{n}}{\partial \mathbf{X}^{n}}\right]+\left[\boldsymbol{\Lambda}_{R}^{n}\right]^{T}\left[\frac{\partial \mathbf{R}^{n}}{\partial \mathbf{X}^{n}}\right]+\left[\boldsymbol{\Lambda}_{R}^{n+1}\right]^{T}\left[\frac{\partial \mathbf{R}^{n+1}}{\partial \mathbf{X}^{n}}\right]+\left[\boldsymbol{\Lambda}_{F}^{n}\right]^{T}\left[\frac{\partial \mathbf{F}^{n}}{\partial \mathbf{X}^{n}}\right]} & =0,
\end{array}
$$

and for $n=0$,

$$
\begin{array}{rlrl}
\text { Load : } & & {\left[\frac{\partial g}{\partial \mathbf{f}^{0}}\right]+\left[\boldsymbol{\Lambda}_{F}^{0}\right]^{T}\left[\frac{\partial \mathbf{F}^{0}}{\partial \mathbf{f}^{0}}\right]+\sum_{k=1}^{N}\left[\boldsymbol{\Lambda}_{G}^{k}\right]^{T}\left(\left[\frac{\partial \mathbf{G}^{k}}{\partial \overline{\mathbf{T}}_{r}^{k}}\right]\left[\frac{\partial \overline{\mathbf{T}}_{r}^{k}}{\partial \mathbf{f}^{0}}\right]+\left[\frac{\partial \mathbf{G}^{k}}{\partial \mathbf{T}_{s}^{k}}\right]\left[\frac{\partial \mathbf{T}_{s}^{k}}{\partial \mathbf{f}^{0}}\right]\right)} & =0, \\
\text { Flow : } & {\left[\frac{\partial g}{\partial \mathbf{Q}^{0}}\right]+\left[\boldsymbol{\Lambda}_{R}^{0}\right]^{T}\left[\frac{\partial \mathbf{R}^{0}}{\partial \mathbf{Q}^{0}}\right]+\left[\boldsymbol{\Lambda}_{R}^{1}\right]^{T}\left[\frac{\partial \mathbf{R}^{1}}{\partial \mathbf{Q}^{0}}\right]+\left[\boldsymbol{\Lambda}_{F}^{0}\right]^{T}\left[\frac{\partial \mathbf{F}^{0}}{\partial \mathbf{Q}^{0}}\right]} & =, \\
\text { Grid : } & {\left[\frac{\partial g}{\partial \mathbf{X}^{0}}\right]+\left[\boldsymbol{\Lambda}_{G}^{0}\right]^{T}\left[\frac{\partial \mathbf{G}^{0}}{\partial \mathbf{X}^{0}}\right]+\left[\boldsymbol{\Lambda}_{R}^{0}\right]^{T}\left[\frac{\partial \mathbf{R}^{0}}{\partial \mathbf{X}^{0}}\right]+\left[\boldsymbol{\Lambda}_{R}^{1}\right]^{T}\left[\frac{\partial \mathbf{R}^{1}}{\partial \mathbf{X}^{0}}\right]+\left[\boldsymbol{\Lambda}_{F}^{0}\right]^{T}\left[\frac{\partial \mathbf{F}^{0}}{\partial \mathbf{X}^{0}}\right]} & =0, \\
\text { Reference Grid : } & {\left[\boldsymbol{\Lambda}_{\hat{G}}\right]^{T}\left[\frac{\partial \hat{\mathbf{G}}}{\partial \hat{\mathbf{X}}}\right]+\left[\boldsymbol{\Lambda}_{G}^{0}\right]^{T}\left[\frac{\partial \mathbf{G}^{0}}{\partial \hat{\mathbf{X}}}\right]} & =0 .
\end{array}
$$

The adjoint equations are solved in the inverse order in time. In the current implementation, $\left[\partial \mathbf{F}^{n} / \partial \mathbf{f}^{n}\right]$ is the identity matrix, thus the load adjoint equation becomes a simple algebraic equation. The summation terms appearing in Eqs. (27) and (30) correspond to the sensitivities of rigid motions and surface elastic motions to sectional airload variations, which are computed via imaginary perturbations of individual components of the airloads $\mathbf{f}^{n}$ at each time level $n$. For each such perturbation, the complex-variable CA solution and the transform matrices, $\mathbf{T}_{r}^{k}$ and $\mathbf{T}_{s}^{k}$, are computed for time levels $k, n \leq k \leq N$. In contrast to the analysis procedure, in which the CA code operates on a single processor, the computations of sensitivities to airload perturbations are distributed to all the processors used in the CFD computation. The total computational time for evaluating the complex-variable sensitivities is proportional to the number of airstations that control the data exchange between the structural and aerodynamic models, the number of time steps $N$, and inversely proportional to the number of processors used in parallel computing. The contributions of structural sensitivities to the CFD flow and grid adjoint solutions are accounted for through the vectors $\left[\mathbf{\Lambda}_{F}^{n}\right]^{T}\left[\partial \mathbf{F}^{n} / \partial \mathbf{Q}^{n}\right]$ and $\left[\boldsymbol{\Lambda}_{F}^{n}\right]^{T}\left[\partial \mathbf{F}^{n} / \partial \mathbf{X}^{n}\right]$ in the flow and grid adjoint equations, respectively. Here, $\left[\partial \mathbf{F}^{n} / \partial \mathbf{Q}^{n}\right]$ and $\left[\partial \mathbf{F}^{n} / \partial \mathbf{X}^{n}\right]$ represent the sectional airloads sensitivities with respect to the conserved flow variables and grid solutions, respectively. A verification test for the sectional airloads sensitivities is provided in Section III.C. At time level $n=0$, the matrices, $\left[\partial \mathbf{F}^{0} / \partial \mathbf{f}^{0}\right],\left[\partial \mathbf{R}^{0} / \partial \mathbf{Q}^{0}\right]$, and $\left[\partial \mathbf{G}^{0} / \partial \mathbf{X}^{0}\right]$, are identity matrices, and the load, flow and grid adjoint equations become simple algebraic equations.

The general form of the sensitivity derivatives of the objective function is written as:

$$
\begin{array}{r}
\frac{d L}{d \mathbf{D}}=\left[\frac{\partial g}{\partial \mathbf{D}}\right]+\left[\boldsymbol{\Lambda}_{R}^{0}\right]\left[\frac{\partial \mathbf{G}^{0}}{\partial \overline{\mathbf{Q}}}\right]\left[\frac{\partial \overline{\mathbf{Q}}}{\partial \mathbf{D}}\right]+\sum_{n=1}^{N}\left[\boldsymbol{\Lambda}_{R}^{n}\right]^{T}\left[\frac{\partial \mathbf{R}^{n}}{\partial \mathbf{D}}\right]+\left[\boldsymbol{\Lambda}_{G}^{0}\right]^{T}\left(\left[\frac{\partial \mathbf{G}^{0}}{\partial \hat{\mathbf{X}}_{s}}\right]\left[\frac{d \hat{\mathbf{X}}_{s}}{d \mathbf{D}}\right]+\left[\frac{\partial \mathbf{G}^{0}}{\partial \overline{\mathbf{T}}_{r}^{0}}\right]\left[\frac{\partial \overline{\mathbf{T}}_{r}^{0}}{\partial \mathbf{D}}\right]\right) \\
+\sum_{n=1}^{N}\left[\boldsymbol{\Lambda}_{G}^{n}\right]^{T}\left(\left[\frac{\partial \mathbf{G}^{n}}{\partial \hat{\mathbf{X}}_{s}}\right]\left[\frac{d \hat{\mathbf{X}}_{s}}{d \mathbf{D}}\right]+\left[\frac{\partial \mathbf{G}^{n}}{\partial \overline{\mathbf{T}}_{r}^{n}}\right]\left[\frac{\partial \overline{\mathbf{T}}_{r}^{n}}{\partial \mathbf{D}}\right]+\left[\frac{\partial \mathbf{G}^{n}}{\partial \mathbf{T}_{s}^{n}}\right]\left[\frac{\partial \mathbf{T}_{s}^{n}}{\partial \mathbf{D}}\right]\right)+\left[\boldsymbol{\Lambda}_{\hat{G}}\right]^{T}\left(\left[\frac{\partial \hat{\mathbf{G}}}{\partial \hat{\mathbf{X}}_{s}}\right]\left[\frac{d \hat{\mathbf{X}}_{s}}{d \mathbf{D}}\right]\right) . \\
7 \text { of } 14
\end{array}
$$


Specifically, the sensitivity with respect to flow design parameters (e.g., angle of attack) is computed as

$$
\frac{d L}{d \mathbf{D}}=\left[\frac{\partial g}{\partial \mathbf{D}}\right]+\left[\boldsymbol{\Lambda}_{R}^{0}\right]\left[\frac{\partial \mathbf{G}^{0}}{\partial \overline{\mathbf{Q}}}\right]\left[\frac{\partial \overline{\mathbf{Q}}}{\partial \mathbf{D}}\right]+\sum_{n=1}^{N}\left[\boldsymbol{\Lambda}_{R}^{n}\right]^{T}\left[\frac{\partial \mathbf{R}^{n}}{\partial \mathbf{D}}\right] ;
$$

the sensitivity with respect to shape design parameters is computed as

$$
\frac{d L}{d \mathbf{D}}=\left[\frac{\partial g}{\partial \mathbf{D}}\right]+\sum_{n=1}^{N}\left[\boldsymbol{\Lambda}_{G}^{n}\right]^{T}\left[\frac{\partial \mathbf{G}^{n}}{\partial \hat{\mathbf{X}}_{s}}\right]\left[\frac{d \hat{\mathbf{X}}_{s}}{d \mathbf{D}}\right]+\left[\boldsymbol{\Lambda}_{\hat{G}}\right]^{T}\left[\frac{\partial \hat{\mathbf{G}}}{\partial \hat{\mathbf{X}}_{s}}\right]\left[\frac{d \hat{\mathbf{X}}_{s}}{d \mathbf{D}}\right] ;
$$

the sensitivity with respect to kinematics design parameters (e.g., rotation rate) is computed as

$$
\frac{d L}{d \mathbf{D}}=\left[\frac{\partial g}{\partial \mathbf{D}}\right]+\left[\boldsymbol{\Lambda}_{G}^{0}\right]^{T}\left[\frac{\partial \mathbf{G}^{0}}{\partial \overline{\mathbf{T}}_{r}^{0}}\right]\left[\frac{\partial \overline{\mathbf{T}}_{r}^{0}}{\partial \mathbf{D}}\right]+\sum_{n=1}^{N}\left[\boldsymbol{\Lambda}_{G}^{n}\right]^{T}\left(\left[\frac{\partial \mathbf{G}^{n}}{\partial \overline{\mathbf{T}}_{r}^{n}}\right]\left[\frac{\partial \overline{\mathbf{T}}_{r}^{n}}{\partial \mathbf{D}}\right]+\left[\frac{\partial \mathbf{G}^{n}}{\partial \mathbf{T}_{s}^{n}}\right]\left[\frac{\partial \mathbf{T}_{s}^{n}}{\partial \mathbf{D}}\right]\right) .
$$

\section{Numerical Results}

This section provides verification of the current implementation of loose and tight coupling procedures for CFD/CA systems by comparing the solutions for a test model of the baseline HART-II rotorcraft configuration ${ }^{7,35}$ with established benchmark computations and experimental data. The complex-variable analysis is verified by comparisons with relevant real-valued, finite-difference computations. The adjoint formulation for sectional airloads computation is verified with the complex-variable approach. Sensitivities of the complete multidisciplinary CFD/CA computations for lift and drag functions to the angle-of-attack design parameter are presented.

\section{A. HART-II Rotorcraft Analysis}

\section{CFD/CA Loose Coupling Simulations}

The flow conditions in the HART-II baseline case include a tip Mach number of 0.6387, an advance ratio of 0.151, and a shaft tilt angle of $4.5^{\circ}$ (adjusted after the wind tunnel wall correction). This case represents a descending flight, where blade-vortex interactions are significant. Other important variables for the blade geometry and test conditions are detailed elsewhere. ${ }^{7}$ Solutions for the HART-II baseline case were obtained with an unstructured mixed-element mesh containing approximately 14 million nodes and are compared to established solutions of FUN3D/CAMRADII (NL-2) ${ }^{6}$ and a previous implementation of FUN3D/DYMORE (GIT-1) ${ }^{7}$ as well as experimental data. In the present study, the BDF2 ${ }_{\text {opt }}$ temporal scheme is used to advance the flow computation in time with a time step corresponding to the increment of $1^{\circ}$ azimuth angle. In ten coupling cycles that involve 5.5 revolutions, the variations in the control angles and thrust deltas are within $0.01^{\circ}$ and $0.1 \%$, respectively. Figure 2 illustrates vortex structures near the HARTII rotor in the baseline case, where the BVI phenomena and the formation of blade tip vortices are clearly observed. Converged solutions of aerodynamic loading and blade tip motions are examined in the following.

Figures 3(a) and (b) demonstrate comparisons of the normal force and pitching moment at the $87 \%$ blade radial station evaluated from the current approach (F/D), NL-2, and GIT-1 solutions, and the measurement data. The magnitudes and shapes of the normal force predicted by the current F/D solution are in good agreement with those of the NL-2 solutions. Furthermore, the events of BVI at the advancing side and particularly the retreating side are captured well by the F/D analysis. The under prediction between $5^{\circ}$ and $40^{\circ}$ and the over prediction between $230^{\circ}$ and $270^{\circ}$ appearing in the GIT-1 solution are not present in the current F/D simulation. The differences observed here are possibly due to the fact that the present implementation of F/D coupling procedures follows closely with those of the FUN3D/CAMRADII development to date, while the GIT-1 solution was obtained with an earlier version of FUN3D, where differences exist in the global slicing procedures for extracting the blade sectional airloads and the conversions of elastic motions to the blade surface geometries. A similar comparison is made for the aerodynamic pitching moment, where the solutions of F/D, NL-2 and GIT-1 methods are generally in a good agreement, particularly in the retreating side.

Figure 4 compares the azimuthal variation of elastic motions including flap, lead-lag and torsion deformations at the blade tip. The flap deflections are obtained by removing the pre-cone angles $\left(2.5^{\circ}\right)$ from the vertical displacements, while the elastic torsion is obtained by excluding the pre-twist angles and the pitch control inputs from the total geometric pitch angle. As demonstrated in Fig. 4(a), the overall characteristics of the predicted blade tip flap deflections in the F/D solution agree well with those of the NL-2 solution, both in magnitude and phase. Compared 


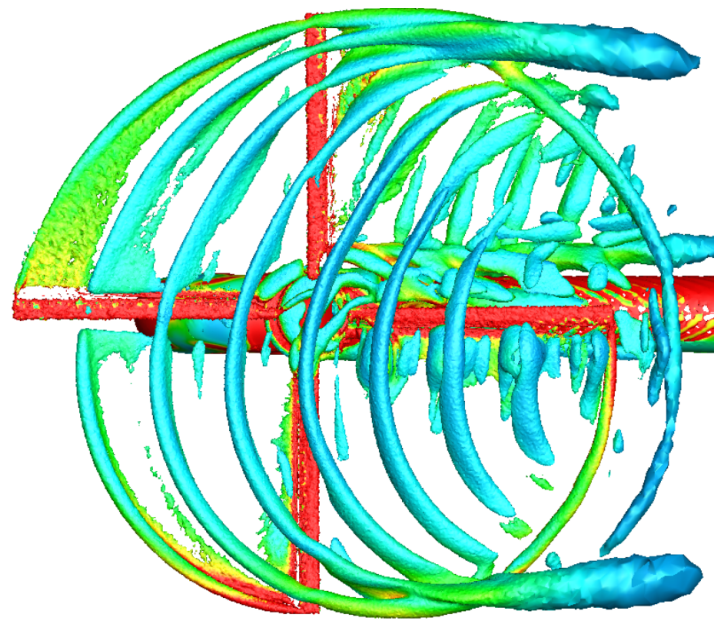

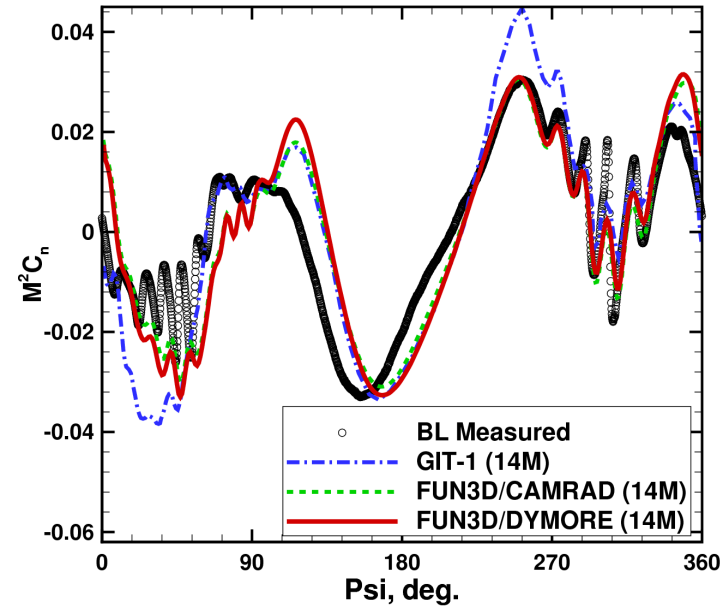

(a) Normal force prediction

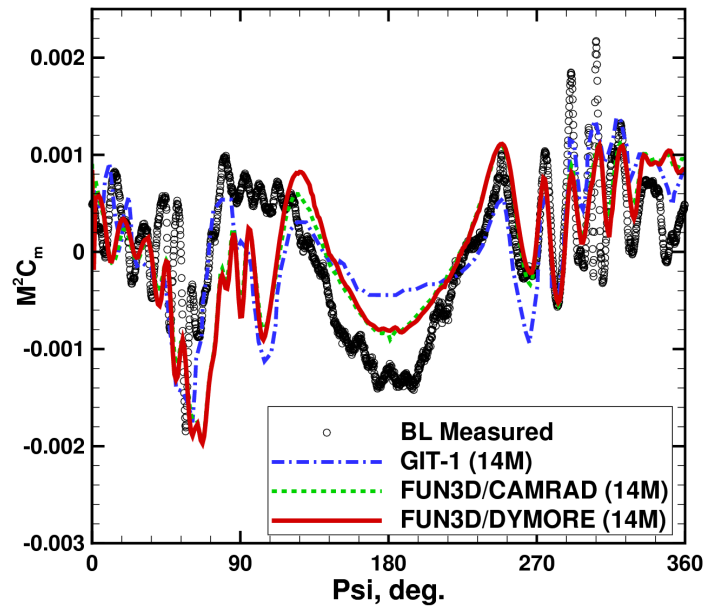

(b) Pitch moment prediction

Figure 3. Comparisons of normal force and pitching moment predictions at $87 \%$ radial station with means removed for the baseline HART-II rotor.

to the GIT-1 solution, some differences can be observed, especially in the first and third quadrants of the rotor disk. These discrepancies are likely caused by the differences in the airload predictions as observed in Figs. 3(a)-(b). All the computational results exhibit a secondary elastic flap maximum at approximately $40^{\circ}-50^{\circ}$ azimuth, while the magnitude of the current solution is higher than that of the GIT-1 result but quite similar to that of the NL-2 solution. Blade tip lead-lag deflections are evaluated in Fig. 4(b), where consistent agreement between motions of the F/D and NL-2 solutions is again achieved. Compared to the measurement data, a constant offset roughly amounting to $1 / 5$ of the chords in the elastic lead-lag deflections is also observed, but this translational offset behavior is commonly seen in other work. ${ }^{36,37}$ Figure 4(c) compares the elastic torsion at the blade tip, where the 2/rev behavior of the elastic twist is predicted well in the F/D solution. In addition, the overall torsion levels are more accurately captured by the F/D solution as compared to the GIT-1 solution, which shows approximately $1^{\circ}$ under predictions across all azimuth locations.

\section{CFD/CA Tight Coupling Simulations}

The tight coupling F/D algorithm is verified in this section by comparing tight and loose coupling simulations conducted with the same trim control angles. Trim computations are generally performed with a loose coupling CFD/CA approach. To achieve converged trim solutions, the CA model typically simulates many revolutions per each coupling 


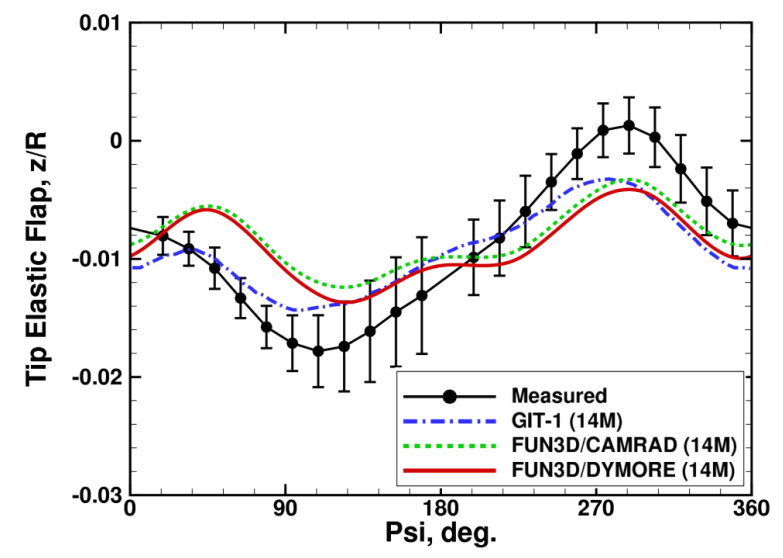

(a) Flap deflections relative to pre-cone $\left(2.5^{\circ}\right)$

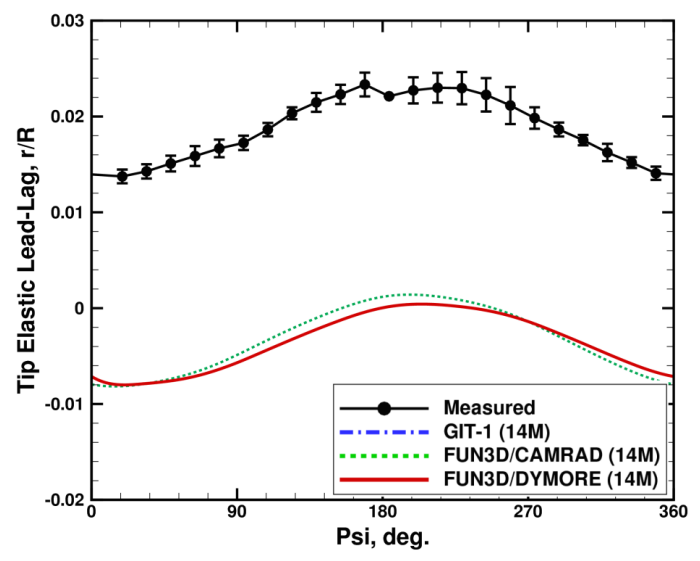

(b) Lead-lag deflections

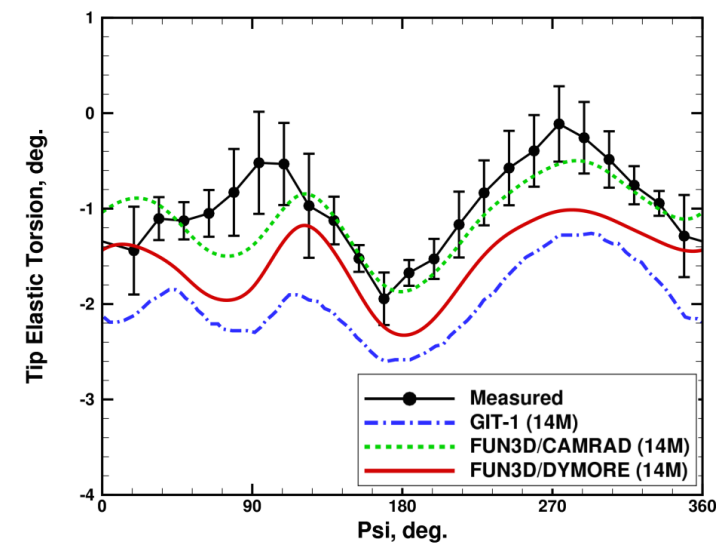

(c) Torsion deflections relative to pre-twist and control angles

Figure 4. Predictions of blade tip flap, lead-lag and torsion deflections for the baseline HART-II rotor.

iteration, which becomes expensive for a tight coupling procedure. In the present work, the trim control angles are taken from a fully converged loose coupling solution. The tight coupling simulations then start from the loose coupling solution, and the collective and cyclic pitch control angles prescribed by the loose coupling trimmed solutions are kept throughout the tight coupling computation. Such a tight coupling procedure should result in minimal change in the solutions.

Figure 5 shows comparisons between the loose and tight coupling F/D solutions for predictions of the normal force and pitch moment at $87 \%$ of the radial station, and the tip flap deflections in the HART-II baseline rotorcraft test case. One can observe that the tight and loose coupling procedures deliver almost identical solutions, which implies that the tight coupling F/D solver functions correctly, and the rotor maintains the trimmed condition.

\section{B. Complex-Valued CFD/CA Analysis}

Complex-variable sensitivities for the coupled CFD/CA analysis can be obtained by operating both models in fully complex modes and perturbing the imaginary part of a design parameter. One such computation can be used to obtain the sensitivities of multiple objective functions with respect to a single design parameter. To ensure that the complexvalued DYMORE model functions properly, real parts of the complex-valued DYMORE solutions describing the linear and angular displacements at the quarter-chord line of a rotor blade in the HART-II baseline rotorcraft test case are examined. The examination shows that the real parts of the DYMORE solutions are identical (matching all significant digits) to those obtained by the real-valued DYMORE model.

Table 1 lists aerodynamic lift at time level $n=3$, computed by the real-valued F/D tight coupling procedures and by the complex-valued F/D tight coupling procedures with several perturbed design parameters including angle of attack and shape design parameters on different blade surfaces of the HART-II baseline rotorcraft configuration. The real parts of the lift function in the complex analyses agree with that computed by the real-valued F/D analysis to machine 


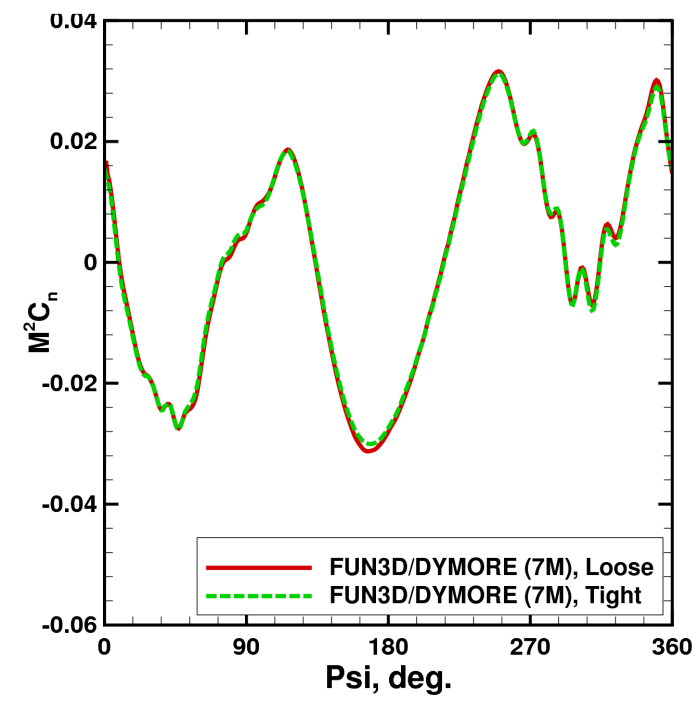

(a) Normal force prediction

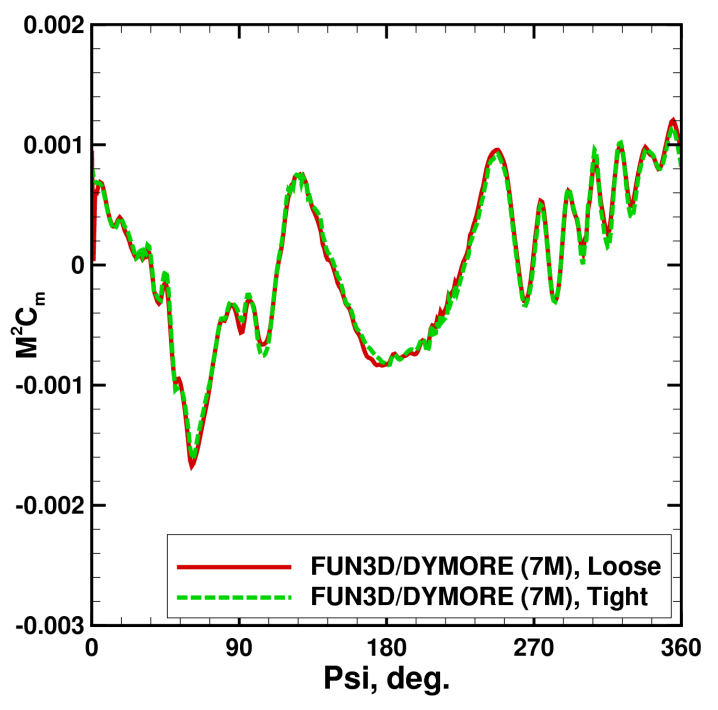

(b) Pitch moment prediction

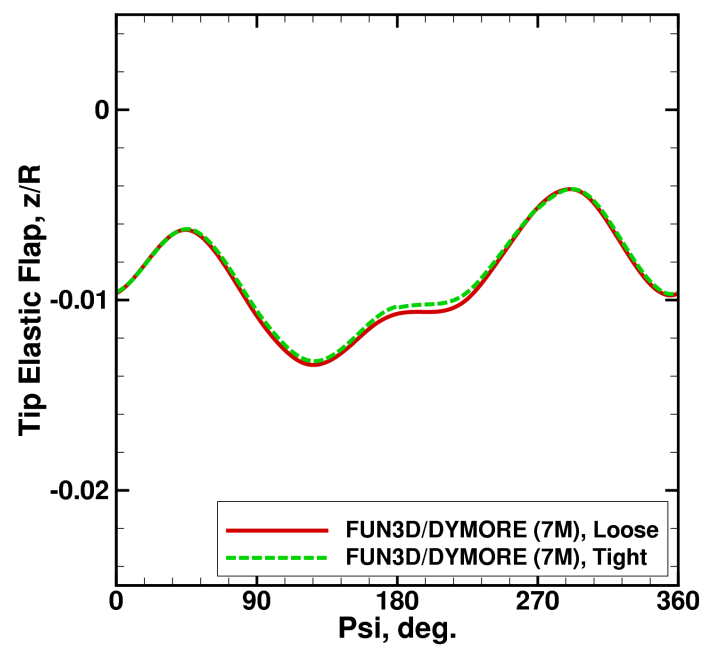

(c) Blade tip deflections

Figure 5. Comparisons of normal force and pitching moment predictions at $87 \%$ radial station with means removed for the baseline HART-II rotor with tight and loose coupling approaches on a $7 \mathrm{M}$ node unstructured mesh.

Table 1. Real-valued and complex F/D analyses for lift (at time level 3) in the baseline HART-II rotorcraft test case.

\begin{tabular}{cccr}
\hline Analysis & Perturbed parameters & Real part of solution & \multicolumn{1}{c}{ Sensitivity } \\
\hline Real & & 0.000798918217192 & \\
& Angle of attack & 0.000798918217192 & 0.000024054292434 \\
Complex & Shape variable, blade 1 & 0.000798918217192 & -0.000044901491848 \\
& Shape variable, blade 2 & 0.000798918217192 & 0.000009593294576 \\
& Shape variable, blade 3 & 0.000798918217192 & -0.000000293789365 \\
& Shape variable, blade 4 & 0.000798918217192 & 0.000005815724362 \\
\hline
\end{tabular}

zero. The imaginary parts of the objective functions in the complex analyses correspond to the lift sensitivities with respect to the specified design parameters and can be used for verification of the multidisciplinary CFD/CA sensitivity analysis. 
Table 2. Comparisons of sectional airloads sensitivities with respect to the grid coordinates ( $y$-component) and conserved flow variables $(x$-component of momentum) at $70 \%$ radial station via direct differentiation and complex-variable approaches.

\begin{tabular}{|c|c|c|c|}
\hline Method & $\partial F_{x} / \partial Q$ & $\partial F_{y} / \partial Q$ & $\partial F_{z} / \partial Q$ \\
\hline Adjoint & 0.000041123560446 & 0.000126949885201 & -0.006184933414864 \\
\hline Complex variable & 0.000041123560446 & 0.000126949885201 & -0.006184933414864 \\
\hline Methods & $\partial F_{m x} / \partial Q$ & $\partial F_{m y} / \partial Q$ & $\partial F_{m z} / \partial Q$ \\
\hline Adjoint & -0.000035570041307 & -0.000005662910700 & -0.000000350458662 \\
\hline Complex variable & -0.000035570041307 & -0.000005662910700 & -0.000000350458662 \\
\hline Methods & $\partial F_{x} / \partial X$ & $\partial F_{y} / \partial X$ & $\partial F_{z} / \partial X$ \\
\hline Adjoint & -1.66200987502660 & 1.97008265283363 & 0.520855236517486 \\
\hline Complex variable & -1.66200987502690 & 1.97008265283398 & 0.520855236517574 \\
\hline Methods & $\partial F_{m x} / \partial X$ & $\partial F_{m y} / \partial X$ & $\partial F_{m z} / \partial X$ \\
\hline Adjoint & -0.015116700097615 & -0.015298667011763 & 0.007316138436884 \\
\hline Complex variable & -0.015116700097618 & -0.015298667011765 & 0.007316138436883 \\
\hline
\end{tabular}

Table 3. Sensitivities of lift and drag to the angle-of-attack design parameter evaluated by the real-valued central difference (CD) methods with perturbation sizes of $10^{-6}$ and $10^{-9}$, complex-variable approach with perturbation size of $10^{-50}$, and the coupled F/D adjoint sensitivity analysis approach.

\begin{tabular}{ccc}
\hline Approach & Lift sensitivity & Drag sensitivity \\
\hline Real-valued CD,$h=10^{-6}$ & 0.000024054294350 & 0.000015909433903 \\
Real-valued CD,$h=10^{-9}$ & 0.000024047540541 & 0.000015921931245 \\
Complex variable, $h=10^{-50}$ & 0.000024054292434 & 0.000015909425336 \\
Multidisciplinary adjoint & 0.000024054286300 & 0.000015909424183 \\
\hline
\end{tabular}

\section{Coupled CFD/CA Sensitivity Computation}

The complex-variable method is employed to compute sensitivities of structural responses with respect to aerodynamic loads and relevant design parameters. All other derivatives required for the evaluation of the adjoint variables and the final sensitivity are implemented by hand differentiation of the corresponding routines in the CFD/CA system. In this section, a verification test for sectional airloads sensitivities with respect to shape variables and conserved flow variables is performed via the complex-variable approach for the HART-II rotorcraft test example discussed in Section III.A. Table 2 shows comparisons of representative aerodynamic airloads derivatives at $70 \%$ radial station computed by the hand differentiation and complex-variable approaches for a given state of viscous flow and grid solutions. As observed, the hand-differentiated sensitivities agree with complex-variable sensitivities well; at least 12-digit agreement is obtained, which is considered as a sufficiently good agreement. ${ }^{25}$

The sensitivities of aerodynamic lift and drag functions evaluated at time level $n=3$ with respect to the angle of attack is computed by the multidisciplinary F/D sensitivity analysis that integrates adjoint-based sensitivities of FUN3D and F/D interfaces with complex-variable sensitivities of DYMORE (cf. Eq. (35)). Although only the flow adjoint solutions are required in the evaluation of the sensitivity to a flow design parameter, the grid adjoint solutions must also be computed due to the coupling. For verification, all the time-dependent problems in both forward and adjoint analyses are converged to machine zero residuals. For demonstration purpose, the first-order backward difference temporal scheme is employed in this example; other higher-order temporal schemes available in FUN3D will be tested in the future work.

Table 3 lists the sensitivities of lift and drag at time level $n=3$ to the angle-of-attack design parameter computed by the real-valued finite difference (second-order central difference) methods with perturbation sizes of $10^{-6}$ and $10^{-9}$, respectively, the complex-variable approach with a perturbation size of $10^{-50}$, and the multidisciplinary F/D sensitivity analysis. Compared to the complex-variable sensitivity, the real-valued finite-difference results corresponding to the perturbation size of $10^{-6}$ show good accuracy in the estimations of lift and drag sensitivities, while the accuracy degrades significantly as the smaller perturbation size is selected. This loss of accuracy of the finite-difference derivatives with small perturbation size is expected due to subtractive cancellation errors, and highlights the shortcomings of real-valued finite differences. The multidisciplinary F/D sensitivities show good agreement with the complex-variable results; approximately 11 digit match is achieved in both lift and drag sensitivities. 


\section{Conclusions}

This paper has presented a multidisciplinary sensitivity analysis framework for high-fidelity rotorcraft computations conducted by a tightly coupled multidisciplinary system including a computational fluid dynamics code, FUN3D, and a rotorcraft comprehensive analysis code, DYMORE. The implementation of the current FUN3D/DYMORE (F/D) analysis procedures has been verified by simulating the baseline HART-II test model. The computed solutions show a good agreement with established predictions of unsteady aerodynamic airloads and various elastic deflections. Discretely-consistent adjoint formulations have been developed and verified for F/D interfaces. Structure-related sensitivities of deforming and rigid motions of component grids are evaluated through a complex-variable approach. The complex-variable method verifies sensitivity derivatives computed with the adjoint-based methods. Complex-variable implementation of sensitivity analysis of DYMORE and the coupled F/D system is verified by comparing with realvalued analysis and sensitivities. Results show that the blade sectional airload derivatives computed by the adjointbased and complex-variable methods are in good agreement. Sensitivities of the lift and drag functions obtained by complex-variable F/D simulations are compared with sensitivities computed by the multidisciplinary sensitivity analysis, which couples adjoint-based flow and grid sensitivities of FUN3D and F/D interfaces with complex-variable sensitivities of DYMORE structural responses. Future work will be concentrated on the verification of the coupled F/D multidisciplinary sensitivity analysis approach for all types of design parameters and applying it to gradient-based multidisciplinary optimization of rotorcraft configurations.

\section{Acknowledgments}

The work was supported by the NASA Revolutionary Vertical Lift Technology Project contract NNL15AB93T. The authors would like to thank Dr. Marilyn Smith at Georgia Institute of Technology for providing the initial FUN3D/DYMORE interface routines as well as the DYMORE input deck for the HART-II configuration. The authors would also like to acknowledge the support of the HART II partners from the U.S. Army Aero-Flight Dynamics Directorate (AFDD), German Aerospace Center (DLR), German-Dutch Windtunnel (DNW), and French Aeronautics and Space Research Center (ONERA).

\section{References}

\footnotetext{
${ }^{1}$ Bauchau, O., Bottasso, C., and Nikishkov, Y., "Modeling Rotorcraft Dynamics with Finite Element Multibody Procedures," Mathematical and Computer Modeling, Vol. 33, 2001, pp. 1113-1137.

${ }^{2}$ Johnson, W., Rotorcraft Aeromechanics, Cambridge Aerospace Series, Cambridge University Press, 2013.

${ }^{3}$ Saberi, H., Khoshlahjeh, M., Ormiston, R. A., and Rutkowski, M. J., "Overview of RCAS and Application to Advanced Rotorcraft Problems," American Helicopter Society 4th Decennial Specialists Conference on Aeromechanics.

${ }^{4}$ Johnson, W., "A History of Rotorcraft Comprehensive Analyses," NASA/TP-2012-216012, April 2012, Ames Research Center, Moffett Field, CA.

${ }^{5}$ Altmikus, A. and Wagner, S., "On the Timewise Accuracy of Staggered Aeroelastic Simulations of Rotary Wings," AHS Aerodynamics, Acoustics, and Test and Evaluation Specialist Meeting, San Francisco, CA, 2002.

${ }^{6}$ Biedron, R. T. and Lee-Rausch, E., "Rotor Airloads Prediction Using Unstructured Meshes and Loose CFD/CSD Coupling," AIAA Paper 2008-7341, June 2008.

${ }^{7}$ Smith, M. J., Lim, J. W., van der Wall, B. G., Baeder, J. D., Biedron, R. T., Boyd Jr., D. D., Jayaraman, B., Jung, S. N., and Min, B.-Y., "The HART II International Workshop: An Assessment of the State of the Art in CFD/CSD Prediction," CEAS Aeronautical Journal, Vol. 4, 2013, pp. 345-372.

${ }^{8}$ Celi, R., "Recent Applications Of Design Optimization To Rotorcraft - A Survey," Journal of Aircraft, Vol. 36, 1999, pp. $176-189$.

${ }^{9}$ Walsh, J. L., Young, K. C., Tarzanin, F. J., Hirsh, J. E., and Young, D. K., "Optimization Issues with Complex Rotorcraft Comprehensive Analysis," AIAA Paper 1998-4889, Sept. 1998.

${ }^{10}$ Murthy, T. S., "Design Sensitivity Analysis of Rotorcraft Airframe Structures for Vibration Reduction," NASA CP 2457, NASA Langley Research Center Sensitivity Analysis in Engineering, 1986, pp. 299-318.

${ }^{11}$ Mishra, A., Mani, K., Mavriplis, D., and Sitaraman, J., "Time Dependent Adjoint-based Optimization for Coupled Fluid-Structure Problems," Journal Computational Physics, Vol. 292, July 2015, pp. 253-271.

${ }^{12}$ Wang, L., Mavriplis, D. J., and Anderson, W. K., "Adjoint Sensitivity Formulation for Discontinuous Galerkin Discretizations in Unsteady Inviscid Flow Problems," AIAA Journal, Vol. 48, No. 12, 2010, pp. 2867-2883.

${ }^{13}$ Wang, L. and Anderson, W. K., "Shape Sensitivity Analysis for the Compressible Navier-Stokes Equations via Discontinuous Galerkin Methods," Computers \& Fluids, Vol. 69, 2012, pp. 93-107.

${ }^{14}$ Nielsen, E. J. and Diskin, B., "Discrete Adjoint-Based Design for Unsteady Turbulent Flows on Dynamic Overset Unstructured Grids," AIAA Journal, Vol. 51, No. 6, 2013, pp. 1355-1373.

${ }^{15}$ Newman III, J. C., Anderson, W. K., and Whitfield, D. L., "Multidisciplinary Sensitivity Derivatives Using Complex Variables," Computational Fluid Dynamics Laboratory, NSF Engineering Research Center for Computational Field Simulation, MSSU-COE-ERC-98-08, 1998.

${ }^{16}$ Fabiano, E., Mavriplis, D., and Sitaraman, J., "Adjoint-based Aeroacoustics Design Optimization for Blade Vortex Interaction Noise," AIAA Paper 2015-1801, Jan. 2015.
} 
${ }^{17}$ Fabiano, E. and Mavriplis, D., “Adjoint-based Aeroacoustics Design Optimization of Flexible Rotors in Forward Flight," AHS Forum 72, West Palm Beach, FL, May 2016.

${ }^{18}$ Corliss, G., Faure, C., Griewantk, A., Hascoet, L., and Naumann, U., Automatic differentiation of algorithms: from simulation to optimization, Springer-Verlag, 2002.

${ }^{19}$ Aubert, P., Cesare, N., and Pironneau, O., "Automatic Differentiation in C++ Using Expression Templates and Application to a Flow Control Problem," Computing and Visualization in Science, Vol. 3, 2001, pp. 197-208.

${ }^{20}$ Martins, J. R. R. A. and Hwang, J. T., "Review and Unification of Methods for Computing Derivatives of Multidisciplinary Computational Models," AIAA Journal, Vol. 51, No. 11, 2013, pp. 2582-2599.

${ }^{21}$ Anderson, W. K. and Bonhaus, D. L., "An Implicit Upwind Algorithm for Computing Turbulent Flows on Unstructured Grids," Computers \& Fluids, Vol. 23, No. 1, 1994, pp. 1-21.

${ }^{22}$ Biedron, R. T., Carlson, J.-R., Derlaga, J. M., Gnoffo, P. A., Hammond, D. P., Jones, W. T., Kleb, B., Lee-Rausch, E. M., Nielsen, E. J., Park, M. A., Rumsey, C. L., Thomas, J. L., and Wood, W. A., "FUN3D Manual: 12.9,” NASA-TM-2016-219012, 2016.

${ }^{23}$ Noack, R., Bogar, D., Kunz, R., and Carrica, P., "SUGGAR++: An Improved General Overset Grid Assembly Capability," AIAA Paper 2009-3992, June 2009.

${ }^{24}$ Spalart, P. and Allmaras, S., “A one-equation Turbulence Model for Aerodynamic Flows,” Le Recherche Aerospatiale, Vol. 1, 1994 , pp. 5-21.

${ }^{25}$ Nielsen, E. J. and Diskin, B., "Discrete Adjoint-Based Design for Unsteady Turbulent Flows on Dynamic Overset Unstructured Grids," AIAA J., Vol. 51, No. 6, 2013, pp. 1355-1373.

${ }^{26}$ Nielsen, E. J., “Adjoint-Based Aerodynamic Design of Complex Aerospace Configurations,” ASME 2016-7573, 2016.

${ }^{27}$ Roe, P. L., "Approximate Riemann Solvers, Parameter Vectors, and Difference Schemes," Journal Computational Physics, Vol. 43, No. 2, 1981, pp. 357-372.

${ }^{28}$ van Leer, B., "Towards the Ultimate Conservative Difference Scheme, V. A Second Order Sequel to Godunov's Method," J. Com. Phys., Vol. 32, No. 1, 1979, pp. 101-136.

${ }^{29}$ Burg, C. O. E., "Higher Order Variable Extrapolation For Unstructured Finite Volume RANS Flow Solvers,” AIAA Paper $2005-4999,2005$.

${ }^{30}$ Vatsa, V., Carpenter, M. H., and Lockard, D., "Re-evaluation of An Optimized Second Order Backward Difference (BDF2OPT) Scheme for Unsteady Flow Applications," AIAA Paper 2010-0122, Jan. 2010.

${ }^{31}$ Noack, R., "DiRTlib: A Library to Add an Overset Capability to Your Flow Solver," AIAA Paper 2005-5116, June 2005.

${ }^{32}$ Abras, J. N., Lynch, C. E., and Smith, M. J., "Computational Fluid Dynamics-Computational Structural Dynamics Rotor Coupling Using an Unstructured Reynolds-Averaged Navier-Stokes Methodology," Journal of The American Helicopter Society, Vol. 57, No. 012001, 2012 , pp. 1-14.

${ }^{33}$ Potsdam, M., Yeo, H., and Johnson, W., "Rotor Airloads Prediction Using Loose Aerodynamic/Structural Coupling," The American Helicopter Society 60th Annual Forum, 2004.

${ }^{34}$ Nielsen, E. J. and Anderson, W. K., "Recent Improvements in Aerodynamic Design Optimization on Unstructured Meshes," AIAA J., Vol. 40, No. 6, 2002, pp. 1155-1163.

${ }^{35}$ van der Wall, B. G., "A Comprehensive Rotary-Wing Data Base for Code Validation: The HART II International Workshop," The Aeronautical Journal, Vol. 115, No. 1164, 2011, pp. 91-102.

${ }^{36}$ Kumar, A., Viswamurthy, S. R., and Ganguli, R., "Correlation of Helicopter Rotor Aeroelastic Response with HART II Wind Tunnel Test," 34th European Rotorcraft Forum, 2008, Liverpool, UK.

${ }^{37}$ Lim, J.-W., "An Assessment of Rotor Dynamics Correlation for Descending Flight using CSD/CFD Coupled Analysis," 64th Annual AHS Forum, 2008, Montreal, Canada. 